\title{
Erratum to: Quantitative Imaging Biomarker Ontology (QIBO) for Knowledge Representation of Biomedical Imaging Biomarkers
}

\author{
Andrew J. Buckler • Tiffany Ting Liu • Erica Savig •
}

Baris E. Suzek • Daniel L. Rubin • David Paik

Published online: 17 May 2013

(C) Society for Imaging Informatics in Medicine 2013

Erratum to: J Digit Imaging

DOI 10.1007/s10278-013-9599-2

This article published with an incorrect author list due to an inadvertent error in the correction cycle. The correct and complete list of authors and affiliations in this paper are:

Andrew J. Buckler, Tiffany Ting Liu, Erica Savig, Baris

E. Suzek, Daniel L. Rubin, David Paik

The publisher regrets the error.

The online version of the original article can be found at http://dx.doi.org/ 10.1007/s10278-013-9599-2.

A. J. Buckler $(\square)$

BBMSC, 225 Main Street, Suite 15,

Wenham, MA 01984, USA

e-mail: andrew.buckler@bbmsc.com

URL: www.bbmsc.com

T. T. Liu $\cdot$ D. L. Rubin $\cdot$ D. Paik

Biomedical Informatics Training Program, Stanford University,

Stanford, CA 94305, USA

T. T. Liu $•$ D. L. Rubin $\cdot$ D. Paik

Department of Radiology, Stanford University, Stanford,

CA 94305, USA

E. Savig

Cancer Biology Program, Stanford University, Stanford,

CA 94305, USA

B. E. Suzek

Georgetown University, Washington, DC 20007, USA 